\title{
Constraining the pass-band of future space-based coronagraphs for observations of solar eruptions in the FeXIV 530.3 nm "green line"
}

\author{
Alessandro Bemporad ${ }^{1}$ (D) Paolo Pagano ${ }^{2}$. \\ Silvio Giordano ${ }^{1} \cdot$ Silvano Fineschi $^{1}$
}

Received: 4 August 2016 / Accepted: 26 May 2017 / Published online: 15 June 2017

(C) Springer Science+Business Media Dordrecht 2017

\begin{abstract}
Observations of the solar corona in the FeXIV $530.3 \mathrm{~nm}$ "green line" have been very important in the past, and are planned for future coronagraphs on-board forthcoming space missions such as PROBA-3 and Aditya. For these instruments, a very important parameter to be optimized is the spectral width of the band-pass filter to be centred over the "green line". Focusing on solar eruptions, motions occurring along the line of sight will Doppler shift the line profiles producing an emission that will partially fall out of the narrower pass-band, while broader pass-band will provide observations with reduced spectral purity. To address these issues, we performed numerical (MHD) simulation of CME emission in the "green line" and produced synthetic images assuming 4 different widths of the pass-band $(\Delta \lambda=20 \AA, 10 \AA$, $5 \AA$, and $2 \AA$ ). It turns out that, as expected, during solar eruptions a significant fraction of "green line" emission will be lost using narrower filters; on the other hand these images will have a higher spectral purity and will contain emission coming from parcels of plasma expanding only along the plane of the sky. This will provide a better definition of single filamentary features and will help isolating single slices of plasma through the eruption, thus reducing the problem of superposition of different features along the line of sight and helping physical interpretation of limb events. For these reasons, we suggest to use narrower band passes $(\Delta \lambda \leq 2 \AA)$ for the observations of solar eruptions with future coronagraphs.
\end{abstract}

Electronic supplementary material The online version of this article (doi:10.1007/s10686-017-9545-2) contains supplementary material, which is available to authorized users.

Alessandro Bemporad

bemporad@oato.inaf.it

1 INAF-Turin Astrophysical Observatory, via Osservatorio 20, 10025 Pino Torinese, Italy

2 School of Mathematics and Statistics, University of St. Andrews, North Haugh, St. Andrews, Fife, Scotland, UK 
Keywords Instrumentation: Coronagraphs $\cdot$ Methods: Numerical $\cdot$ Sun: Corona $\cdot$ Sun: Coronal mass ejections

\section{Introduction}

The solar corona is the tenuous and high temperature atmosphere of the Sun, made up with a magnetized plasma. One of the main hurdles in the observations of the corona is the fact that at all wavelengths its brightness is several orders of magnitude smaller than the brightness of the solar disk. For this reason, the design of any coronagraph is driven by the main requirement not only to reject the light coming directly from the Sun, but also to reduce as much as possible the level of illumination coming indirectly from the Sun and entering in the instrument via multiple diffractions and reflections, the socalled "stray light".

For this reason the best conditions for observations of the solar corona are occurring during the fascinating natural spectacle of total solar eclipses, when the disk of the Moon is perfectly occulting the Sun, having almost exactly the same angular dimensions of it. Nevertheless, total solar eclipses occur only in rare occasions, often in quite remote places on Earth, and have a very short duration by a few minutes. Ground-based observations of the corona are also provided by internally occulted coronagraphs (such as the famous Lyot coronagraph), but with a field of view (FOV) limited only to the inner part of the corona, where its brightness overcome that of the sky. Moreover, ground-based coronagraphs are limited by the night and day alternation, local weather conditions, and are restricted only to the visible and near infrared pass-bands, where our atmosphere is almost transparent.

The only solution to overcome all these difficulties is to carry out a coronagraph into space. Many successful space-based instruments acquired coronagraphic observations from different satellites orbiting around the Earth (e.g. on-board the Solar Maximum Mission - SMM; [3]), around the Sun-Earth Lagrangian point L1 (e.g. on-board the Solar and Heliospheric Observatory - SOHO; [7]) or around the Sun (e.g. on-board the twin Solar \& Terrestrial Relationship Observatory - STEREO; [11]). The majority of these instruments were and are externally occulted coronagraphs (e.g. SOHO/LASCOC2 and C3, [4]; STEREO/COR1 and COR2, [27]), with the advantage to have a significant reduction of light scattered inside the instrument, but requiring distant and large occulters mounted on long and delicate external booms preventing the observations of the inner part of the solar corona. On the other hand, some of these instruments were internally occulted coronagraphs (e.g. SOHO/LASCO-C1), with the advantage to have a much inner view of the corona provided by solid single object telescopes, but with a reduced signal-to-noise ratio due to the increased light scattered inside the instrument.

A challenging solution will be provided in the next future by the ASPIICS coronagraph (Association of Spacecraft for Polarimetric and Imaging Investigation of the Corona of the Sun; [21]) on-board the PROBA-3 mission, the third one of the ESA PROBA (Project for On-Board Autonomy) satellite series. For this innovative coronagraph one satellite will carry the telescope, while the occultation of the Sun will be provided by an external occulter mounted on a second satellite and maintained in fine formation flying, with both satellites orbiting around the Earth. With an inter-satellite 
distance on the order of $150 \mathrm{~m}$, and a formation flying maintained with different sensors within a precision of fractions of millimetres, ASPIICS will be the largest externally-occulted coronagraph ever launched. This will allow to have a FOV covering not only the outer, but also the inner corona (as usually provided only by internally occulted instruments), and at the same time to have a very small fraction of stray light (as provided only by externally occulted instruments). Fine formation and then coronagraphic observations will occur over a period of six hours per orbit, corresponding to the passage around the apogee of a highly eccentric elliptical orbit with period of slightly less than $20 \mathrm{~h}$. During formation flying the instrument projected FOV will cover heliocentric distances from $3.0 \mathrm{R}_{\text {sun }}$ down to $1.08 \mathrm{R}_{\text {sun }}$ thus allowing an unprecedented view of the early evolution of solar dynamic events (Coronal Mass Ejections - CMEs and flares), and other related or unrelated phenomena such as shock waves excitations, acceleration of Solar Energetic Particles, waves propagation, solar wind acceleration, and others.

To this end, the ASPIICS coronagraph will observe the solar corona with multiple filters selecting different pass-bands; one of them will be centred on one of the brightest coronal emission lines in the visible spectral range, the FeXIV $530.3 \mathrm{~nm}$ line, also known as the famous "green line". Historically, lot of observations of this line have been acquired in the past decades from ground-based instruments, in particular during total solar eclipses, but only a very few dynamic events were observed in this line at that time. Early ground-based detections of "coronal transient events", "flare spray", "hemispherical shells" were observed in the green line at Sacramento Peak Observatory since the end of " 50 s (Fig. 1). Many of these events were correlated with flares, $\mathrm{H} \alpha$ limb activity and radio burst $[6,8,16]$. These early observations demonstrated that "green line events" were correlated with solar activity cycle, but these events were observed even before the idea of "Coronal Mass Ejection" came up with the Skylab mission in the years ' $70 \mathrm{~s}$.

Later on many eruptions were detected in the green-line from other ground-based coronagraphs such as the NOrikura Green-Line Imaging System (NOGIS - Japan, a $10 \mathrm{~cm}$ coronagraph with tunable birefringent filter with passband of $1 \AA$, FOV 1.03-
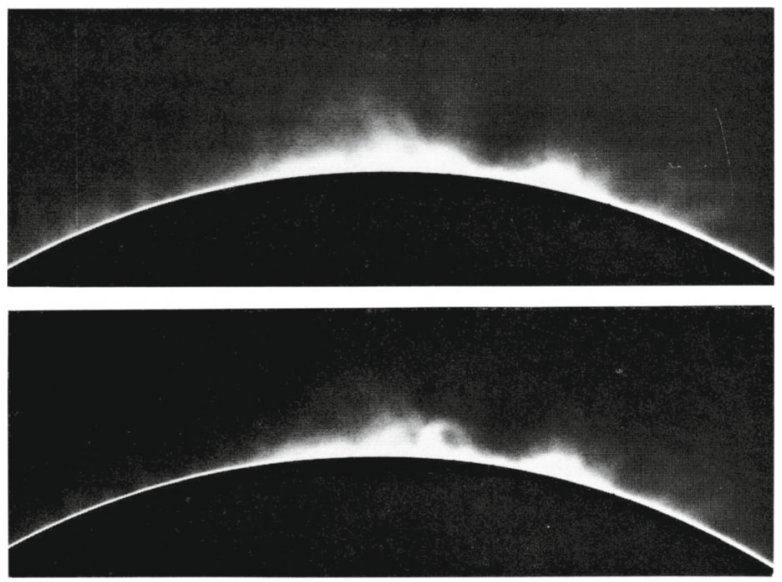

Fig. 1 Example of a "fast transient event" observed in the "green line" at Sac Peak Coronagraph in 1967; top and bottom frames were separated by seven minutes of time (from [6]) 
$\left.1.33 \mathrm{R}_{\text {sun }}\right)($ e.g. $[10,26])$ and the Mirror Coronagraph for Argentina (MICA - Argentina, FOV 1.05-2.0 $\mathrm{R}_{\text {sun }}$, coronagraphs with Fabry-Perot interferometer with passband by $1.5 \AA$; e.g. $[2,25])$. These observations provided unique information on the CME source regions and initiation processes, and also suggested that the green line emission could be used as a good tracer of the unknown coronal magnetic field topology. Nevertheless, all these observations had the limitations mentioned above with ground-based instruments.

Only one space-based coronagraph ever observed CMEs in the green line: the LASCO-C1 instrument on-board SOHO. LASCO-C1 was an internally occulted coronagraph, with FOV going from 1.1 to $3.2 \mathrm{R}_{\text {sun }}$ provided by a Fabry-Perot interferometer with passband by $0.5 \AA$. This instrument observed many eruptive events (Fig. 2) showing "for the first time an unambiguous connection between coronal green-line transients and CMEs observed in Thomson scattered white light in the outer corona" and that "fast (speed $>25 \mathrm{~km} / \mathrm{s}$ ) green line events appear to be related to the prominence core of the CME, rather than the leading front observed in whitelight"[19]. Also LASCO-C1 observations demonstrate that "large-scale streamer structure follows the magnetic pattern fairly precisely" [24], suggesting again the usefulness of this line to derive information on the coronal field topology. These observations also showed the limits achievable with internally occulted coronagraphs.

Now, thanks to the forthcoming PROBA-3, a new era of CME observations is fast approaching. Nevertheless, in order to obtain new scientific results, the optimization of instrumental parameters will be, as always, of fundamental importance. One of the main parameters to be optimized for ASPIICS observations of CMEs is the spectral width of the "green line" pass-band, as it will be explained in the next Sections. For this reason, the work described here focused on MHD simulations of "green line" CME emission as will be observed by ASPIICS assuming different band-pass filters. In particular in this work we address this problem by synthesizing the FeXIV emission from a MHD simulation of a flux rope ejection and then testing how different bandpass filters would perform on these synthesized images. A similar approach has proven to be useful to simulate the analysis of white light coronagraphic images that will be acquired by the Metis coronagraph [1, 9, 22] on-board the forthcoming ESA Solar Orbiter mission [15]. After a short description of the problem (Section 2) and of the numerical simulations we performed (Section 3), our results will be described (Section 4) and we will give our discussion and conclusions (Section 5).

\section{Optimizing the band-pass filter for CME observations}

In order to constrain the pass-band of the "green-line" filter it is necessary to take into account two opposite requirements. In white light, the coronal intensity is due to spectroscopic line emission (the E-corona, related with atomic emission processes), superimposed onto the continuum emission (the K-corona and the F-corona, due respectively to scattering and reflection of photospheric light from coronal electrons and interplanetary dusts). Hence, a measurement $G$ of the "green line" intensity requires also to measure the intensity $K$ and $F$ of the other emissions to be subtracted. In order to isolate the line emission with respect to the background continuum, it is necessary to combine exposures acquired in-band (i.e. centred on the spectral line) with those acquired out-band 

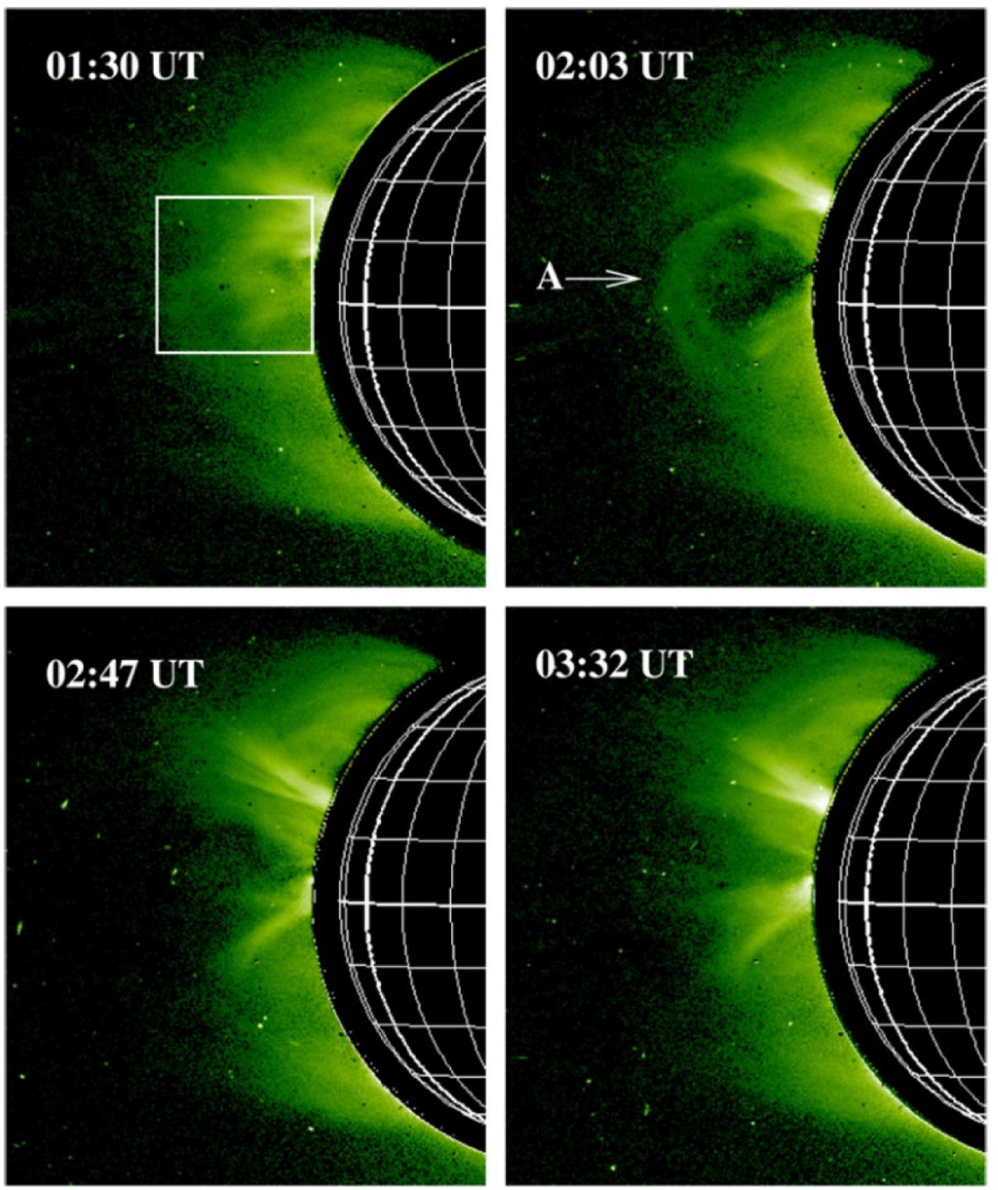

Fig. 2 Example of a sequence acquired by the LASCO-C1 coronagraph showing a solar eruption (top panels) and the post-CME coronal reconfiguration (bottom panels) observed in the "green line" emission (from [19])

(i.e. in the nearby continuum). A fundamental parameter is then the so-called "spectral purity" $P(\%)$ of a coronal line, that we define here as $P_{G}=G /(G+K+F)$, and represents the percentage of line emission with respect to the total coronal emission.

The spectral purity is related with the width $\Delta \lambda_{F}(\AA)$ of pass-band of the instrument filter. By decreasing the width of the pass-band it is possible to have a higher spectral purity of the line, and thus to have a better determination of the line intensity, as we will also show here. On the other hand, during a solar eruption, a fraction of the emitting plasma could have a significant velocity along the line of sight (LOS), emitting Doppler shifted profiles. These shifted profiles will fall partially out of the interval $\Delta \lambda_{\mathrm{F}}$ covered by the filter, thus reducing the measured intensity of the emission corona. Hence, by increasing the width of the pass-band it is possible to observe also Doppler shifted emission coming from solar dynamic phenomena such as CMEs, that would be missed with a very narrow pass-band.

To better quantify these problems, let us assume for instance that the Fe XIV $530.3 \mathrm{~nm}$ spectral line has a Gaussian profile with typical FWHM $\Delta \lambda_{L}=1.0 \AA$ 
(corresponding to thermal motions by $34 \mathrm{~km} / \mathrm{s}$ or kinetic effective temperature by $3.9 \cdot 10^{6} \mathrm{~K}$ ), as measured by previous authors (see e.g. [14]) and let us consider 4 possible values for the FWHM of the Gaussian instrument filter pass-band: $\Delta \lambda_{F 1}=20 \AA, \Delta \lambda_{F 2}=10 \AA, \Delta \lambda_{F 3}=5 \AA$, and $\Delta \lambda_{F 4}=2 \AA$. Estimates of the green line spectral purity $P_{G}$ can be derived by assuming typical values for the $G, K$ and $F$ coronal intensities at different heliocentric distances referring to typical coronal streamer or coronal hole regions. To this end, we assumed values for the $K$ and $F$ coronal intensities (erg $\mathrm{cm}^{-2} \mathrm{~s}^{-1} \mathrm{sr}^{-1}$ ) and full disk spectral intensities (erg $\mathrm{cm}^{-2} \mathrm{~s}^{-1} \mathrm{sr}^{-1} \AA^{-1}$ ) provided by Cox [5] at different altitudes for the minimum of solar activity cycle. Absolute values for the $G$ coronal intensity in the "green line" are more uncertain and quite sparse in the literature. Hence, for the aims of this work, Fe XIV $530.3 \mathrm{~nm}$ coronal intensities have been computed theoretically using the CHIANTI spectral code (v. 7.1; [12]) and considering both the collisional and radiative excitations. These two components have been estimated in stationary coronal structures starting from electron density and temperature radial profiles provided by Vásquez et al. [28] for both the equatorial and polar regions. Moreover, in order to test the possible consequences during the observation of a $\mathrm{CME}$, the "green line" spectral purity $P_{G}$ has been also computed at fixed heliocentric distance of 1.2 solar radii, with the same $K, F$ and $G$ coronal intensities as in the previous case, but by assuming Doppler shifts in the "green line" Gaussian profile due to LOS motions, occurring with speed from 0 up to $500 \mathrm{~km} \mathrm{~s}^{-1}$.

Resulting values of spectral purity $P_{G}$ at different heliocentric distances in the solar corona are shown in Fig. 3 (top panels) for a coronal streamer (left) and a coronal hole (right) by assuming 4 different values of the filter pass-band given above. These plots clearly show that $P_{G}$ decreases in general by increasing $\Delta \lambda_{F}$ (as expected) both in a coronal streamer and a coronal hole at all heliocentric distances. Hence, considering stationary coronal structures, a better measurement of the "green line" intensity would be provided by a narrower pass-band. On the other hand, as it is also shown in Fig. 3 (bottom panels), for dynamic phenomena the situation is reversed. In particular, as the LOS speed increases, because of spectral line Doppler shift, the resulting spectral purity $P_{G}$ drops down much faster for narrower pass-band; hence, considering solar eruptions, these plots seems to suggest that a better measurement of the "green line" intensity would be provided by a broader pass-band, maintaining a more constant spectral purity independently on the LOS speed.

The above results show why it is not easy to choose the best compromise for the width of the band-pass filter. Hence, in what follows we describe how, to solve the problem, the spectroscopic "green line" emission due to an expanding solar eruption has been simulated and then convolved with different pass-bands to provide expected CME images that will be acquired by future instrumentations such as ASPIICS onboard PROBA-3 satellites.

\section{Setting up the MHD simulation}

The simulation of coronagraphic emission due to a CME in a single spectral line is not an easy task for many different reasons. The emission observed in each pixel of a coronagraphic image comes from the integration along the LOS through the optically 

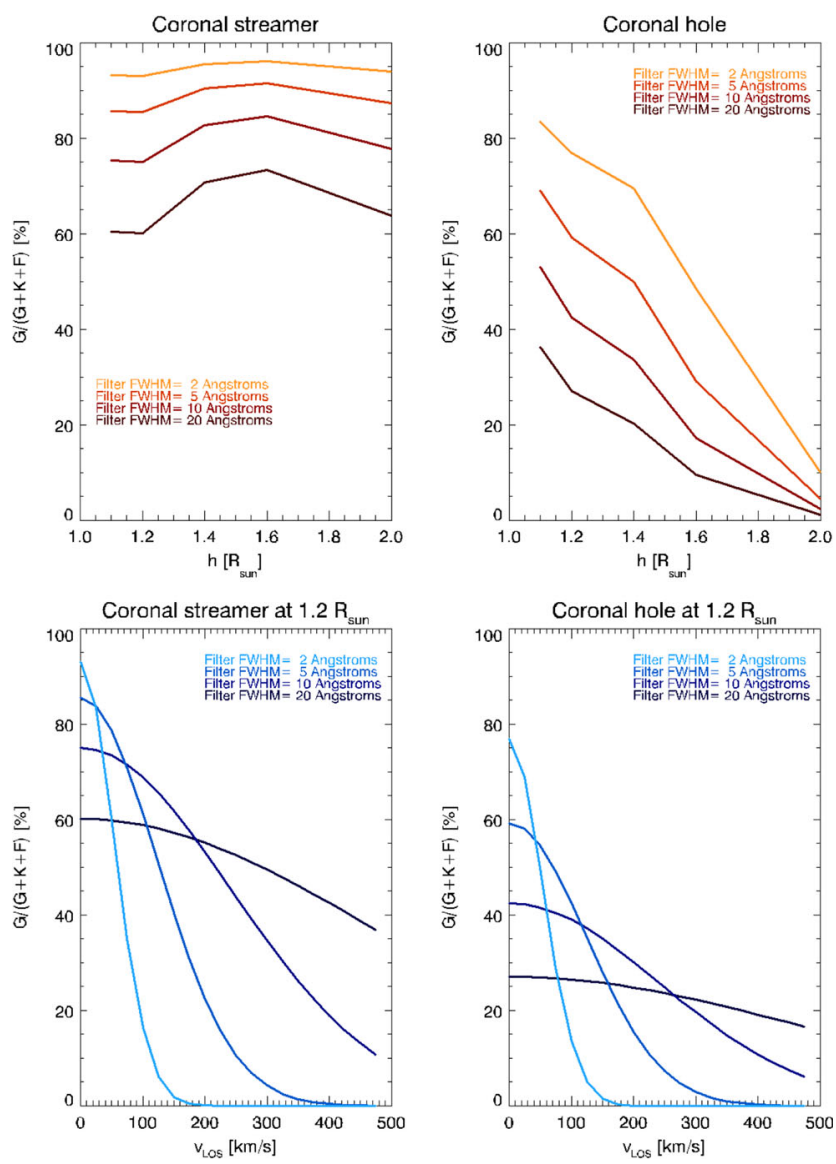

Fig. 3 Top: the "green line" spectral purity as a function of projected heliocentric distances $\left(\mathrm{R}_{\text {sun }}\right)$ computed in a coronal streamer (left) and a coronal hole (right) for different values of the instrument band-pass filter. Bottom: the "green line" spectral purity at constant altitude $\left(1.2 \mathrm{R}_{\text {sun }}\right)$ computed by assuming different values of the LOS speed and different values of the instrument band-pass filter

thin coronal plasma. Hence, it is necessary to start with a $3 \mathrm{D}$ data-cube of plasma parameters (electron density, electron temperature, ion kinetic temperature) to compute the expected emission in each cell aligned with the same LOS. Then, spectroscopic emission from each cell needs to be Doppler shifted taking into account the LOS component of the plasma expansion speed, and then integrated along the LOS to get the synthetic spectrum. The final step consists in the convolution of this spectrum with the assumed shape for the band-pass filter to derive the resulting synthetic "monochromatic" image.

To this end, we started with the sequence of 3D datacubes of plasma parameters obtained with a MHD simulation of a solar eruption, similar to what was done by Pagano et al. [18] to simulate the polarized visible light emission of a CME. In particular, in this work we used the same MHD simulation described by Pagano et al. [17]; in this simulation a flux rope is formed (as described in [13]) embedded 
in realistic coronal density and temperature distributions (as described in [17]). The MHD equations are solved in spherical coordinates with the MPI-AMRVAC software [20] in a simulation domain extending over these intervals: heliocentric distance $r$ from 1 to 3 solar radii, colatitude $\theta$ from $30^{\circ}$ to $100^{\circ}$, and longitude $\varphi$ spanning $90^{\circ}$ (we refer the reader to the above publications for more details on the MHD simulations).

The output time sequence of plasma parameters in $3 \mathrm{D}$ data-cubes is then converted from spherical $(r, \theta, \varphi)$ to Cartesian $(x, y, z)$ coordinates to perform the LOS integration of coronal emission. For each cell along the LOS the input electron density and temperatures have been used first to estimate (again with CHIANTI spectral code) the fraction of $\mathrm{Fe}^{13+}$ ions and then the emissivity of the $\lambda 530.3 \mathrm{~nm}$ "green line", taking into account both collisional (i.e. due to collisions with thermal electrons) and radiative (i.e. due to absorptions of continuum emission) excitations, and by assuming that ionization equilibrium holds over the whole simulation box. The intensity emitted by each cell along the LOS has been used to build a synthetic spectral line profile with Gaussian shape, FWHM determined by assuming that the ion kinetic temperature equals the plasma temperature in each cell given by the MHD simulation, and line centroid $\lambda_{0}$ Doppler shifted by $\Delta \lambda=\lambda_{0}\left(v_{L O S} / c\right)$ where $v_{L O S}$ is the LOS component of the plasma velocity and $c$ is the speed of light. The total line profile expected in each pixel has been derived simply by integrating all the profiles emitted by all the cells aligned along the same LOS. Then, for each pixel the measured total intensity has been determined with the integral of the product between the resulting total line profile and the band-pass filter. This computation has been performed by assuming the same 4 values for the FWHM of the Gaussian band-pass filter mentioned in the previous paragraph $\left(\Delta \lambda_{F 1}=20 \AA, \Delta \lambda_{F 2}=10 \AA, \Delta \lambda_{F 3}=5 \AA\right.$, and $\left.\Delta \lambda_{F 4}=2 \AA\right)$, to determine the effects of broader or narrower pass-bands.

\section{Results}

As an example of this analysis, we show in Fig. 4 the shape of the LOS averaged spectra during the eruption extracted at 3 different pixels in the image. Due to the superposition along the LOS of many different cells where the plasma is expanding towards or away from the observer, the resulting LOS averaged profiles are a superposition of both blue- and red-shifted emissions. The comparison with the shape of the broader $\left(\Delta \lambda_{F 1}=20 \AA\right.$, dashed line $)$ and the narrower $\left(\Delta \lambda_{F 4}=2 \AA\right.$, dotted line) band-pass filters clearly shows that with the narrower filter a significant fraction of the total emission will be lost, because the emission is partially Doppler shifted out of the band-pass filter. On the other hand, the whole "green line" emission will be sampled using the broader band-pass filter. The fraction of total emission that will be lost with the narrower filter is not constant over the image, but is changing pixel by pixel and in time depending on the motions of plasma along the considered LOS. For instance, as shown in Fig. 4, for the specific frame we selected here a small fraction of the emission will be lost in point $\mathrm{C}$, because only a small fraction 
of the spectrum is severely Doppler shifted, contrary to what happens in points $\mathrm{A}$ and $\mathrm{B}$.

As a consequence, images acquired with a narrower band-pass filter will have a higher spectral purity $P_{G}=G /(G+K+F)$, as it is shown clearly in Fig. 5; on the other hand, a significant fraction of the total emission coming from CMEs will be lost, as it is shown in Fig. 6. In particular this Figure shows, for the same frame in the MHD simulation, the ratio between the "green line" intensities $G$ measured with the narrowest ( $2 \AA$ ) and the broadest (20 $\AA$ ) filters (left panel), and the corresponding average centroid $\lambda_{0}$ of the spectrum $s(\lambda)$ computed as $\lambda_{0}=s(\lambda) \lambda d \lambda / s(\lambda) d \lambda$ (right panel). Values of average centroid $\lambda_{0}$ are shown in the range $[-10 \AA,+10 \AA]$ corresponding to the FWHM of the broader band-pass filter considered here $(20 \AA)$. The comparison between these two panels clearly demonstrates that there are two regions in the image where only a small fraction of the emission is lost: 1) coronal regions located out of the $\mathrm{CME}$ in the stationary corona, and 2) coronal regions located inside the CME, and characterized by negligible Doppler shifts, hence negligible LOS motions. Moreover, a comparison between the right panel of Fig. 6 and different panels in Fig. 5 demonstrates that a reduction in the pass-band of the filter allows to significantly recover spectral purity only in the CME regions with small LOS velocities, while for CME regions characterized by larger LOS motions, whose spectroscopic emission is located around the tails of the bandpass filter, an even broader filter pass-band would be required. In fact, assuming a Gaussian band-pass filter with FWHM by $20 \AA$, motions occurring with LOS speed larger than $v_{L O S}=\mathrm{c} \Delta \lambda / \lambda_{0} \sim 565 \mathrm{~km} \mathrm{~s}^{-1}$ will produce a spectroscopic emission that will be reduced by more than a factor 2 .
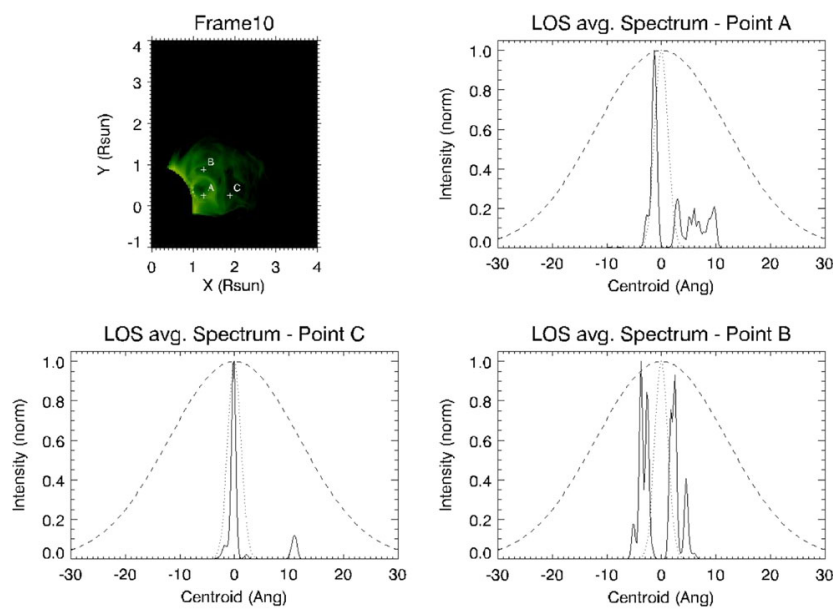

Fig. 4 Top left: example of a simulated coronagraphic image in FeXIV $530.3 \mathrm{~nm}$ "green line" emission. This panel also shows the location of 3 pixels $(\mathrm{A}, \mathrm{B}$ and $\mathrm{C}$ ) in the image where the corresponding LOS averaged spectra are shown in the other 3 panels. Top right, bottom left and right: LOS normalized averaged spectra (solid line) compared with respect to the shape of the $20 \AA$ filter (dashed line) and $2 \AA$ filter (dotted line). A movie showing the evolution during the whole eruption is also available 

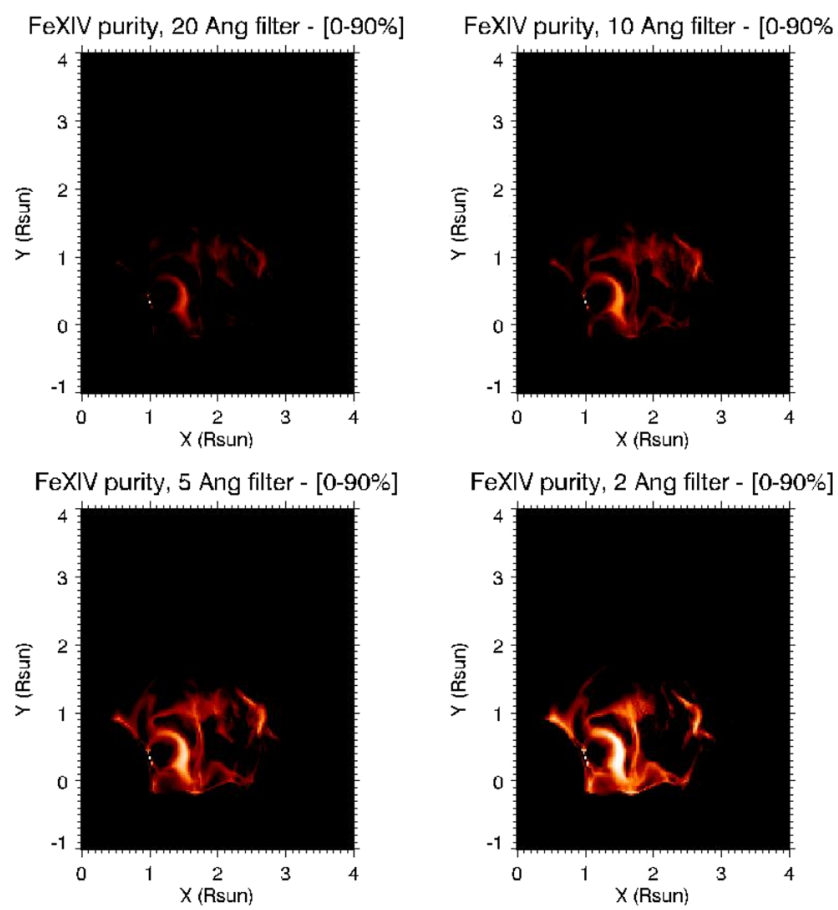

Fig. 5 "green line" spectra purity during the eruption by assuming a filter pass-band with FWHM of $20 \AA$ (top left), $10 \AA$ (top right), $5 \AA$ (bottom left) and $2 \AA$ (bottom right). In all figures the color scale is the same $(0-90 \%)$. A movie showing the evolution during the whole eruption is also available

\section{Discussion and conclusions}

In this work we addressed the problem of defining the better choice for the width of band-pass filters in order to optimize future coronagraphic observations of solar eruptions (CMEs) in the FeXIV $530.3 \mathrm{~nm}$ "green line". To this end, we performed numerical (MHD) simulations of a CME, synthesized "green line" spectral emission, and derived the averaged spectra that will result from the integration along the line of sight (LOS) through the CME. These spectra have been convolved with Gaussian-shaped filters by assuming 4 different values for the pass-band: 2 values for broader-band imaging $(20 \AA$ and $10 \AA$ ) and two values for narrower-band imaging ( $5 \AA$ and $2 \AA$ ).

The results shown here allow us to better understand the differences and similarities in the final "green line" synthetic intensity images (Fig. 7) depending on the width assumed for the Gaussian band-pass filter. In particular, images acquired with the broader filters ( $20 \AA$ and $10 \AA$ ) will capture a larger fraction of the CME emission and then will show many more structures inside the CME; on the other hand, images acquired with the narrower filters $(5 \AA$ and $2 \AA$ ) will miss some of the features associated with the eruption. Nevertheless, images acquired with the broader filters will result in a complex mixing of different filamentary features aligned along the LOS and expanding with different LOS velocities, while images acquired with the narrower filters will isolate the emission coming from the CME features expanding with 

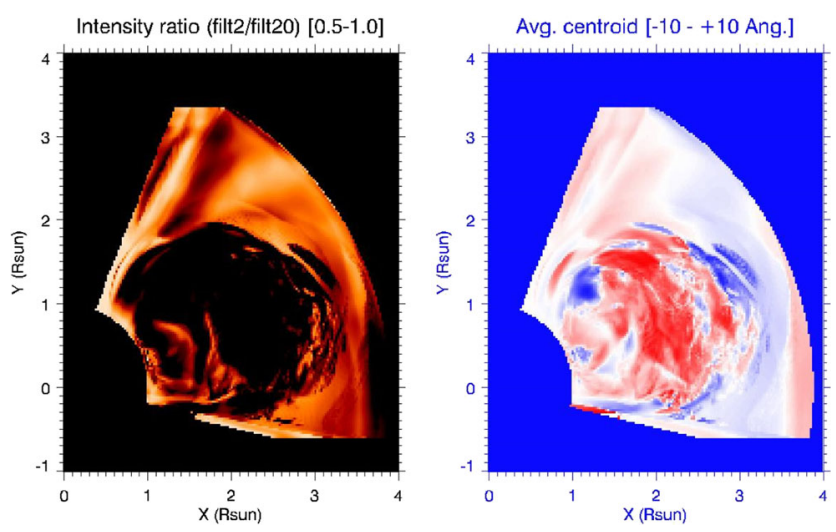

Fig. 6 Left: ratio between the "green line" intensity images sampled with narrower $(2 \AA)$ and the broader (20 A) band-pass filters. Right: distribution of the LOS averaged Doppler shift in the resulting spectrum. A movie showing the evolution during the whole eruption is also available

negligible LOS velocity, hence expanding mainly along the plane of the sky. Also, for the same reasons, in broad band images the CME filamentary structures appear "blurred" with respect to their appearance in narrow band images where the same features have a much better contrast.

This means that images acquired with narrow-band filters show something like a "slice" through the CME plasma, corresponding to the intersection of the 3D CME structure and the 2D plane of the sky. This has very important consequences first for the interpretation of past observations acquired with narrow-band filters such as LASCO-C1 coronagraph $(0.5 \AA)$. In fact, the dark voids observed during and after some of these eruptions (e.g. Fig. 2), very similar to "void" regions shown in our image simulated with the narrower filter (Fig. 7, bottom right panel), were interpreted at that time as regions devoid of emitting plasma. Nevertheless the analysis conducted here demonstrate that this is not the only possible interpretation, because the alternative interpretation could be that those regions were not devoid of plasma, but filled with plasma characterized by a LOS speed larger than what provided by the half-width of the pass-band, hence $v_{L O S}=\mathrm{c}(0.25 \AA) / 5303 \AA \sim 14 \mathrm{~km} \mathrm{~s}^{-1}$. This is a very small LOS velocity easily reached by the coronal plasma during the expansion phase of any CME.

Second, results shown here have very important consequences as well for the selection of pass-bands for future coronagraphic instrumentation dealing with the "green line". As we showed here, narrow-band images of solar eruptions will have at least two advantages:

- a much better spectral purity for CME features expanding along the plane of the sky

- a much better definition of filamentary features expanding along the plane of the sky

One of the main hurdles in the interpretation of coronagraphic observations of CMEs is due to the superposition along the LOS of many different features spatially 

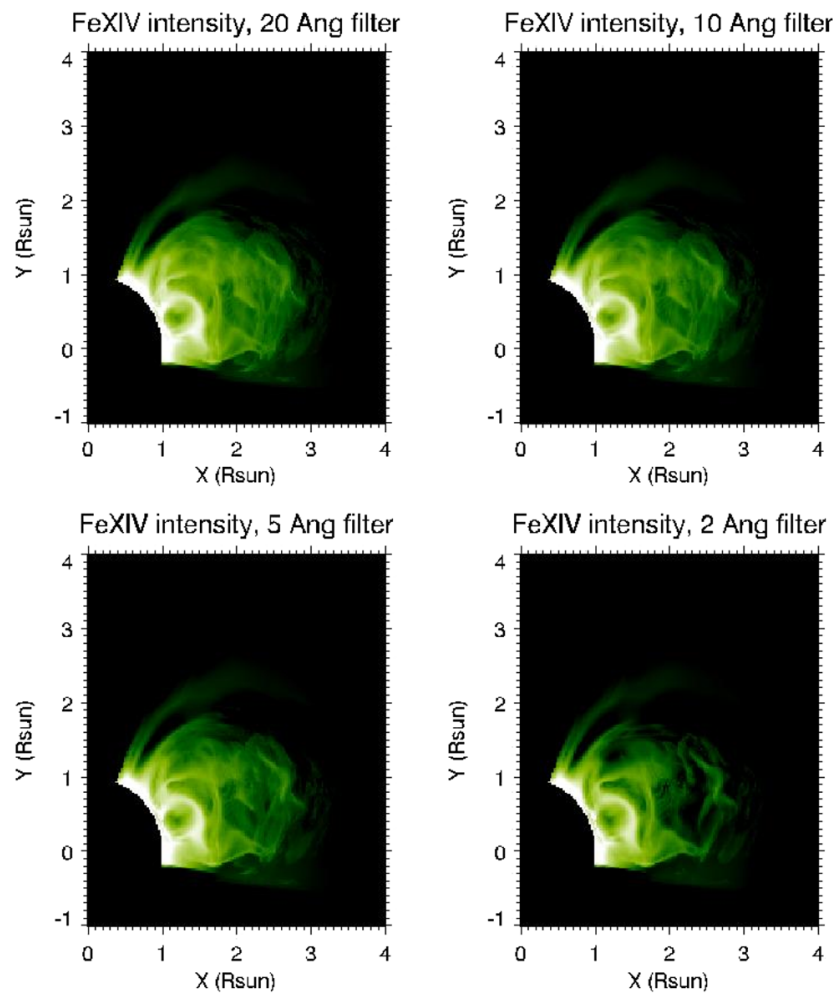

Fig. 7 Comparison between the simulated coronagraphic images of a solar eruption in the "green line" acquired with 4 different band-pass filters. A movie showing the evolution during the whole eruption is also available

located at different points and being mixed all together in the optically thin corona. This superposition very often complicates the determination of plasma physical parameters (such densities and temperatures) inside different parts of the eruption. The above two advantages will help the scientific interpretation of future coronagraphic CME observations in the "green line", helping to isolate single CME features located on the plane of the sky. Hence, the use of narrowband filters is strongly suggested for the study of limb events expanding mainly along the plane of the sky. These results will be very important for future instrumentations that will acquire these kind of images, such as the ASPIICS coronagraph on-board the forthcoming ESA PROBA-3 mission, but also the coronagraph that will fly on-board the Indian Aditya spacecraft [23].

Acknowledgements We acknowledge the use of the open source (gitorious.org/amrvac) MPI-AMRVAC software, relying on coding efforts from C. Xia, O. Porth, R. Keppens. This research has received funding from the European Research Council (ERC) under the European Union's Horizon 2020 research and innovation programme (grant agreement No 647214) and from the UK Science and Technology Facilities Council. The computational work for this paper was carried out on the joint STFC and SFC (SRIF) funded cluster at the University of St Andrews (Scotland, UK). 


\section{References}

1. Antonucci, E., Fineschi, S., Naletto, G., et al.: Multi Element Telescope for Imaging and Spectroscopy (METIS) coronagraph for the Solar Orbiter mission. Proc. of the SPIE, 8443, id. 844309 (2012)

2. Bagalá, L.G., Stenborg, G., Schwenn, R., Haerendel, G.: The eruptive events on September 30, $1998: 1$. The jet. Journal of Geophysical Research. 106(A11), 25239-25248 (2001)

3. Bohlin, J.D., Frost, K.J., Burr, P.T., Guha, A.K., Withbroe, G.L.: Solar maximum mission. Sol Phys. 65(1), 5-14 (1980)

4. Brueckner, G.E., Howard, R.A., Koomen, M.J., et al.: The large angle spectroscopic coronagraph (LASCO). Sol. Phys. 162(1-2), 357-402 (1995)

5. Cox, A. N.: Allen's astrophysical quantities. Publisher: New York: AIP Press; Springer, 2000. Edited by Arthur N. Cox (2000)

6. Demastus, H.L., Wagner, W.J., Robinson, R.D.: Coronal disturbances. I: Fast Transient Events Observed in the Green Coronal Emission Line during the Last Solar Cycle. Solar Physics. 31(2), 449-459 (1973)

7. Domingo, V., Fleck, B., Poland, A.I.: SOHO: the solar and Heliospheric Observatory. Space Sci. Rev. 72(1-2), 81-84 (1995)

8. Dunn, R.B.: Coronal Events Observed in $5303 \AA$. Physics of the Solar Corona, Proceedings of the NATO Advanced Study Institute. Reidel, Dordrecht (1971)

9. Fineschi, S., Antonucci, E., Naletto, G., et al.: METIS: a novel coronagraph design for the Solar Orbiter mission. Proc. of the SPIE, 8443, id. 84433H (2012)

10. Hori, K., Ichimoto, K., Sakurai, T., et al.: Flare-associated coronal disturbances observed with the Norikura green-line imaging System. I. A Coronal Mass Ejection Onset. The Astrophysical Journal. 618(2), 1001-1011 (2005)

11. Kaiser, M.L.: The STEREO mission: an overview. Adv. Space res. 36(8), 1483-1488 (2005)

12. Landi, E., Young, P. R., Dere, K. P., Del Zanna, G., Mason, H. E.: CHIANTI-An Atomic Database for Emission Lines. XIII. Soft X-Ray Improvements and Other Changes. Astrophys J, 763, 2, article id. 86, 9 (2013)

13. Mackay, D.H., van Ballegooijen, A.A.: Models of the large-scale corona. I. Formation, evolution, and liftoff of magnetic flux ropes. Astrophys J. 641(1), 577-589 (2006)

14. Mierla, M., Schwenn, R., Teriaca, L., Stenborg, G., Podlipnik, B.: Analysis of the Fe X and Fe XIV line width in the solar corona using LASCO-C1 spectral data. Astron Astrophys. 480(2), 509-514 (2008)

15. Müller, D., Marsden, R.G., St Cyr, O.C., Gilbert, H.R.: Solar orbiter. Exploring the sun-heliosphere connection. Sol Phys. 285(1-2), 25-70 (2013)

16. Orral, F.Q., Smith, H.J.: The passage of a flare spray through the solar emission corona. Astron. J. 66, 293 (1961)

17. Pagano, P., Mackay, D.H., Poedts, S.: Simulating AIA observations of a flux rope ejection. Astron Astrophys. 568(id.A120), 10 (2014)

18. Pagano, P., Bemporad, A., Mackay, D.H.: Future capabilities of CME polarimetric 3D reconstructions with the METIS instrument: A numerical test. Astron Astrophys. 582(id.A72), 12 (2015)

19. Plunkett, S.P., Brueckner, G.E., Dere, K.P., et al.: The relationship of green-line transients to white-light coronal mass ejections. Sol. Phys. 175(2), 699-718 (1997)

20. Porth, O., Xia, C., Hendrix, T., Moschou, S. P., \& Keppens, R.: MPI-AMRVAC for Solar and Astrophysics. The Astrophysical Journal Supplement Series, Volume 214, Issue 1, article id. 4, 26 pp. (2014)

21. Renotte, E., Alia, A., Bemporad, A., et al.: Design status of ASPIICS, an externally occulted coronagraph for PROBA-3. Proceedings of the SPIE, Volume 9604, id. 96040A 15 pp. (2015)

22. Romoli, M., Landini, F., Antonucci, E., et al.: METIS: the visible and UV coronagraph for Solar Orbiter. Proc. of the ICSO 2014 International Conference on Space Optics (2014)

23. Sankarasubramanian, K.: 31st ASI Meeting, ASI Conference Series, 2013, Vol. 9, pp 43-48 Edited by Pushpa Khare \& C. H. Ishwara-Chandra (2013)

24. Schwenn, R., Inhester, B., Plunkett, S.P., et al.: First view of the extended green-line emission corona at solar activity minimum using the Lasco-C1 coronagraph on SOHO. Sol. Phys. 175(2), 667-684 (1997)

25. Stenborg, G., Schwenn, R., Srivastava, N.: MICA Observations of Coronal Transients. Proceedings of the "8th SOHO Workshop", ESA Special Publications 446. Edited by J.-C. Vial and B. KaldeichSchümann., p. 627 (1999)

26. Suzuki, I., Sakurai, T., Ichimoto, K.: Three-dimensional motion of plasmas associated with a coronal mass ejection observed with NOrikura green-line imaging System (NOGIS). Publications of the Astronomical Society of Japan. 58(1), 165-175 (2006) 
27. Thompson, W.T., Davila, J.M., Fisher, R.R., et al.: COR1 inner coronagraph for STEREO-SECCHI. Innovative telescopes and instrumentation for solar Astrophysics. Edited by Stephen L. Keil, Sergey V. Avakyan. Pro SPIE. 4853, 1-11 (2003)

28. Vásquez, A.M., van Ballegooijen, A.A., Raymond, J.C.: The effect of proton temperature anisotropy on the solar minimum corona and wind. Astrophys J. 598(2), 1361-1374 (2003) 